\title{
Is Genetics Implicated in the Causation of Autism?
}

\section{Samashaptak ${ }^{1}$, Pratnoja Das ${ }^{1}$, Sukanti Bhattacharyya ${ }^{2 *}$ and Tarun Kumar Datta ${ }^{3}$}

${ }^{1}$ Undergraduate Medical Student (MBBS), ICARE Institute of Medical Sciences and Research and Dr. Bidhan Chandra Roy Hospital, Haldia, West Bengal, India

${ }^{2}$ Associate Professor, Department of Medical Physiology, ICARE Institute of Medical Sciences and Research and Dr. Bidhan Chandra Roy Hospital, Haldia, West Bengal, India

${ }^{3}$ Clinical Psychologist, Pavlov Research Institute and Hospital, Kolkata, West Bengal, India

*Corresponding Author: Sukanti Bhattacharyya, Associate Professor, Department of Medical Physiology, ICARE Institute of Medical Sciences and Research and Dr. Bidhan Chandra Roy Hospital, Haldia, West Bengal, India.

DOI: $10.31080 / A S M S .2022 .06 .1194$
Received: December 31, 2021

Published: February 14, 2022

(C) All rights are reserved by Sukanti

Bhattacharyya., et al.

\section{Abstract}

Autism is a neurodevelopmental disorder first introduced by Kanner in 1943. Presently, a group of disorders collectively is considered to be 'Autism Spectrum Disorder' which is an outcome of complex interaction between genes and the environment.

In this investigation, authors considered the historical development of genetic study that revealed the secret of genes responsible for autism. They noted that synapse-related risk genes and susceptibility genes affecting transcription and chromatin-remodelling pathways have an important role behind the mechanism of the disease.

But there are environmental factors also. Currently 40\%-80\% of autism cases are related to some risk genes, but epigenetic, particularly environmental modifiers, like advanced age of father, gestational complications and infections, prenatal exposure to anticonvulsants, or paucity of oestrogen in developmental brain are also playing fundamental roles in heterogeneity of manifestations.

Till date, hundreds of genes have been recognized along with the environmental factors but how they interact to manifest the disease is still unknown due to complexities in interaction and overlap between different neuro-psychiatric disorders.

Presence of second level modifiers has made the situation more multifaceted. However, advancements in sequencing technology, analyzing software, and expansion of databases have made the study of genetic modifiers possible.

Going through the works by various scientists across the globe over the last 75 years, authors have identified a number of chromosomes, multitudes of gene loci, many genetic overlaps, and myriads of modifiers - endocrinal, environmental, and epigenetic. But, at the same time they have warned that though genes play a pivotal role, the other factors also need to be considered and studied in detail with proper attention and finally synthesis all the information reasonably to discover the root cause of autism.

Keywords: Autism; ASD; Neurodevelopmental Disorders; Neuro-psychiatric Disorders; Genetics; Epigenetics

\section{Introduction}

Autism, as first coined by Eugen Bleuler and first described in 1943 by Kanner in his detailed report with thorough patient his- tory of 11 children showing different symptoms of autism, is a neurodevelopmental disorder with impairment of social and communicational behaviours; often narrated as 'mind blindness' in lay media. 
23 years after Kanner, the first epidemiological study estimated to be 4.5 per 10,000 individuals; but increasing awareness and evolvement of Diagnostic and Statistical Manual of Mental Disorders (DSM) criteria, drastically increased it to 1 per 59 individuals with M:F as 4-5:1 [1], but 3:1 [2]. With increase in autism diagnoses, there's a decrease in the number of children considered 'cognitively disabled' or 'learning disabled', suggesting a relabeling of children's disorders.

An Autism Spectrum Disorder (ASD) diagnosis can co-occur with numerous other conditions, due to the broad nature of these definitions; most commonly including motor abnormalities (79\%), gastrointestinal problems (up to 70\%), epilepsy (up to 30\%), intellectual disability (45\%), and sleep disorders (50-80\%) [3]; even with language disorders as per the DSM-IV criteria.

According to the current DSM-5 criteria, only two core features make up an Autism Spectrum Disorder (ASD) diagnosis are persistent deficits in social communication and interaction (SCI) across multiple contexts, and restricted, repetitive patterns of behaviour (RRBs), interests, or activities [3].

Observing monozygotic concordance-prevalent in some families-effect of high paternal age, gestational comorbidities in mothers and even accusing 'refrigerator mother' [4], or relating to the contamination of vaccine with heavy metal like mercury, pathophysiology of autism has long been a wild goose to chase with in the bushes from chromosome to gene loci to epigenetics to environmental influences, so on and so forth. Plethora of overlaps among many neurodevelopmental disorders and neuro-psychiatric illnesses in childhood also makes the issue more complicated to understand. With the help of advanced tools in biotechnology, molecular biology and genetics, like micro array, array-hybridization, SNP, CNV, etc (described in the glossary), instead of unveiling the curtain off compel us to find out the actual causation of autism. In order to put many similar ailments together, an umbrella-term 'Autism Spectrum Disorder' has been coined. ASD, now recognized as a disease of complex interaction between genetics and the environment, has heritability estimating from $40 \%$ to $80 \%$ [5].

Along with extensive genetic studies revealing hundreds of genes linked to autism, epidemiological investigations have begun to illuminate the environmental factors contributing to risk, but how the two interact to contribute to ASD aetiology is still unknown.
Drastically varying phenotypes can be seen in subjects with similar pathogenic variants, as in cases of complex diseases [Cook., et al. 1997; Bolton., et al. 2001]. Factors modulating expression of other genes, i.e. genetic modifiers are likely to exist in individuals having the same pathogenic variant on opposite spectral ends.

\section{Objective of the Study}

In this perplexity, the present study is intended to move across different studies on the subject over last few decades, and to present them in an assertive way with the objective to find out a single common aetiological basis of autism.

\section{Genetics of Autism Spectrum Disorder}

Seeking for candidate ASD genes

Understanding the disease aetiology of ASD, following Kanner's classification of autism, has a paradigm shift from the initial environmental theory to the subsequent genetic theory over the observations of 50x higher incidence among the twins [Folstein., et al. 1977], and 60\% concordance among monozygotic twins against null in dizygotics [Bailey., et al. 1995], risk of ASD having proportional sharing of genome with affected siblings or parents [6-8].

Early karyotypic study focused on the specific gene loci [Gillberg., et al. 1985], like7q, 1p, 3q, 16p, and 15q [7,9,10], was followed by candidate approach to study specific gene families, e.g. Hox family and Wnt gene; but both turned futile [Krebs., et al. 2002; Lamb., et al. 2002; Talebizade., et al. 2002; Zhang., et al. 2002].Since 2001, there was moderate success with findings supporting reelin (RELN), aristaless related homeobox (Arx), methylCpG binding protein 2 (MeCP2), neuroligin 3 (NLGN3), neuroligin 4 (NLGN4), tuberous sclerosis complex 2 (TSC2), and ubiquitin protein ligase E3A (UBE3A)'s involvement in ASD aetiology [Perisco., et al. 2001; Strømme., et al. 2002; Carney., et al. 2003; Jamain., et al. 2003; Serajee et al.,2003; Jiang., et al. 2004].

High Throughput Study (HTS), in early 2000s, yielded genomewide level studies for sequencing of involved genome and found wide variation of genomic involvements in average number of cases, except for few monogenic involvements in specific ASDs like Rett syndrome, fragile $\mathrm{X}$ syndrome, tuberous sclerosis, and Schuurs-Hoeijmakers syndrome $[11,12]$.

Although myriads of genomic study identified hundreds of risk genes, yet majority of the reproducible hits were found having two functional group of proteins: 
- $\quad$ Synapse-related risk genes, encoding cell-adhesion proteins such as neuroligins, neurexins, and cadherins; synaptic vesicle cycling proteins synapsin-1 (SYN1) and synapsin-2 (SYN2); ion transport proteins such as sodium voltage-gated channel alpha subunit 2 (SCN2A), calcium voltage-gated channel subunit alpha1 E (CACNA1E), calcium voltage-gated channel auxiliary subunit beta 2 (CACNB2), potassium voltage-gated channel subfamily Q members 3 and 5 (KCNQ3 and KCNQ5), potassium voltage-gated channel subfamily D member 2 (KCND2), glutamate receptor signalling protein SH3 and multiple ankyrin repeat domains 3 (SHANK3), synaptic Ras GTPase activating protein 1 (SYNGAP1), and gamma-aminobutyric acid type A receptor gamma3 subunit (GABARG3) [13-15]; and de novo mutation of such genes [Turner., et al. 2016; Turner., et al. 2017; Short., et al. 2018].

- Susceptibility genes affecting transcription and chromatinremodelling pathways like MeCP2, UBE3A, chromodomain helicase DNA binding protein 8 (CHD8), activity dependent neuroprotector homeobox (ADNP), pogo transposable element derived with ZNF domain (POGZ), fragile X mental retardation protein (FMRP), and RNA binding forkhead box (RBFOX) genes $[15,16]$.

\section{ASD risk in somatic mosaicism}

Post-zygotic mosaicism is often organ-specific, not ubiquitous in the whole soma, due to of 5\%-7\% de novo variation, which have also been reported to estimate upto $22 \%$ [17], and daily about 5 single nucleotide variants (SNV) during neurogenesis [Bae., et al. 2018; D'Gama., et al. 2018]; are mostly innocuous; but mutation in exon might have serious neurodevelopmental disorder with manifestation of ASD including Rett syndrome, tuberous sclerosis, intellectual disability, schizophrenia, and many other disorders [18,19].

Being progressed from case-based study of ASD [Oliveira., et al. 2003; Sauter., et al. 2003; Papanikolaou., et al. 2006; Havlovicova., et al. 2007; Yurov., et al. 2007; 64; 65; 66], through study on somatic mosaicism explaining 3\%-5\% cases of autism simplex [Freed., et al. 2016; Krupp., et al. 2017], to large cohort-based instrumental study ( $\mathrm{n}=5947$ ) [17] involving Whole Exome-Sequencing (WES) yielded that critical exon-related mosaicism may result in neurodevelopmental abnormalities of either amygdala, related to difficulty in social communication [Rasia-Filho., et al. 2000], or cerebellum, related to gait disorder in autism [Dou., et al. 2017]. Freed and Pevsner through a large wave study $(n=2388)$ of affected families, not only identified ascertainment bias for pathogenic mosaic variations in ASD, but also confirmed implicated candidate gene SCN2A and some more risk genes, and also ascertained the significance of somatic mosaicism in causation of ASD [17].

\section{Contributions of CNVs in susceptibility of ASD}

Only few [20] of either inherited or de novo mutation [Thapar., et al. 2013] in CNV, by duplications, deletions, translocations, and inversions, even stretching several kb, [Marshall., et al. 2008] may result in $10 \%$ of ASD cases [Geschwind, 2011].

Studies on CNV, majorly implicating duplication of $16 \mathrm{p} 11.2$ and alteration in most of the 25 genes there, are related to abnormalities in neuro-development [Blaker-Lee., et al. 2012] and plurality in manifestations due to multigenic involvement, and KCTD 13 (potassium channel tetramerization domain containing 13) is implicated in most of the neuro-psychiatric disorders [Golzio., et al. 2012], might be due to aberration in synaptic transmission though Ras homolog family member A (RHOA) [Escamilla., et al. 2017]. Deletion in 16p11.2 region leading to altered MAP3 kinase activity results in deformed cortical cyto-architecture and reduced brain size [Pucilowska., et al. 2015]. Study on 16p11.2 in Drosophila using RNAi by Iyer (2018) strongly indicated that modifying interactions within CNVs result in the complex phenotypes, not involving any single gene like 16p11.2 [Iyer., et al. 2018].

CNVs in other disease mechanisms are less often studied due to the inadequacy of commonly affected regions. 15q11-13 and 16p11.2, the most prevalent ASD-associated CNVs, are found in roughly $1 \%$ of autism cases [Kumar., et al. 2008; Marshall., et al. 2008; Weiss., et al. 2008; Marshall., et al. 2012], and also there remain no known CNVs with complete penetrance [Marshall., et al. 2008].

Deletions in synaptic genes, such as SHANK3, dipeptidyl peptidase-like 10 (DPP10), neuroligins, and neurexins in autism [21], duplication of CNV in the locus containing the UBE3A gene in autistic traits in mice, and decreased glutamatergic synaptic transmission [Smith., et al. 2011] are also seen in some non-ASD/ schizophrenic controls with dyslexia and dystaxia exhibiting same structural changes [Stefansson., et al. 2014].

A microarray analysis with identified CNVs in ASD-associated genes, showed a positive correlation between duplication size and autism severity, but no correlation with non-verbal IQ. CNV often 
contributes to ASD risk critically with complexity, but there are highly variable phenotypes for similar structural variants in patients.

\section{ASD under epigenetic regulation}

Study with 215 candidate genes demonstrated 19.5\% epigenetic modifier in causation of ASD [22]; similarly another study with risk gene also confirmed it. Phenotypic variance in ASD thus can be explained on the basis of nuclear penetrance of epigenetic modifiers. Twin study demonstrated profound epigenetic modulation of disease phenotype as in 50 pairs of monozygotic twins discordant for ASD reported numerous autism-associated differentially methylated regions, with methylation patterns at some $\mathrm{CpG}$ sites common to symptom groups [Wong., et al. 2014]. KMT2C, lysine methyltransferase 5B (KMT5B), and lysine demethylase 6B (KD$\mathrm{M} 6 \mathrm{~B}$ ); chromatin remodelling proteins including MeCP2, CHD8, and POGZ; RNA-binding/splicing proteins such as FMRP and the RBFOX family, post-translational modification proteins like UBE3A, mindbomb E3 ubiquitin protein ligase 1 (MIB1); or transcription factors like ADNP and additional sex combs like 3 (ASXL3) are the genes with susceptibility loci involving methylation [16]. These are implicated in autism by altered synaptic formation. Mutations in a single epigenetic regulator modifying number of risk genes were studied in depth at 2 key susceptibility genes, MeCP2 and UBE3A. MeCP2 normally regulates synaptic function at the genomic level of GABRB3, brain derived neurotrophic factor (BDNF), distal-less homeobox 5 (DLX5), insulin like growth factor binding protein 3 (IGFBP3), cyclin dependent kinase like 1 (CDKL1), protocadherin beta 1 (PCDHB1), protocadherin 7 (PCDH7), and lin-7 homolog A (LIN7A) [23], but consistently modifies chromatin in ASD patients. Also, there is some alteration in the rate limiting regulation of Glutamergic synapse formation by MeCP2 in ASD pathology [Chao., et al. 2010].

UBE3A (an E3 ubiquitin protein ligase), lying in 15q11-13 and being duplicated in autism, epigenetically reduce excitatory synaptic transmission and is having positively correlated dose-dependent effect on delay of first word and psychomotor regression [Guffanti., et al. 2011; Smith., et al. 2011; Xu., et al. 2018]. UBE3A and one of its substrate proteasome 26S, non-ATPase 4 (Rpn10), leads to pathological alteration of dendritic fine outgrowth in autism [Hamilton., et al. 2012; Puram., et al. 2013]. Several studies on large scale have found different epigenetic regulation in ASD like alteration in histone, acetylome wide association [Sun., et al. 2016].
Altered methylation in the proline rich transmembrane protein 1 (PRRT1) 3' UTR, promoter regions of tetraspanin 32 (TSPAN32), and C11orf21 in cortical tissue, and also in succinate dehydrogenase complex flavoprotein subunit A pseudogene 3 (SDHAP3), in cerebellar tissue [Ladd-Acosta., et al. 2014]; dysregulated miRNAs related to oxytocin receptor (OXTR) gene [Behnia., et al. 2015]. Abnormal cerebellar Purkinje growth due to epigenetic alteration in engrailed homeobox 2 (EN2) [James., et al. 2013]. These risk genes with epigenetic functions and their substrates are the focus of present day therapeutics, e.g. inhibition of FMRP target bromodomain containing 4 (BRD4) in alleviating some of the disease manifestations [Korb., et al. 2017].

\section{Overlapping of ASD risk genes with other diseases}

Cross-Disorder Group of the Psychiatric Genomics Consortium (PGC) in 2013 conducted a very large scale epidemiologic study with 33332 cases and 27888 controls and found multiple cross-overlaps in the risk genes, e.g. inter-alpha-trypsin inhibitor heavy chain 3 (ITIH3), arsenite methyltransferase (AS3MT), calcium voltage-gated channel subunit alpha1 C (CACNA1C), and CACNB2, amounting to genome-wide significance in several neuro-psychiatric disorders, like ASD, schizophrenia, bipolar disorder, ADHD (OR AD/HD), and major depressive disorder [24,25]; having strong correlation with structural variants in loci of dedicator of cytokinesis 8 (DOCK8) and $\mathrm{KN}$ motif and ankyrin repeat domains 1 (KANK1), and phenotypic variations in these disorders [Glessner., et al. 2017]. Schork et al. (2019) [26] after using GWAS to identify susceptibility loci in genes including phosphodiesterase 1A (PDE1A), protein phosphatase 1 regulatory inhibitor subunit $1 \mathrm{C}$ (PPP1R1C), RHOA, immunoglobulin superfamily member 11 (IGSF11), and sortilin related VPS10 domain containing receptor 3 (SORC3), recently hypothesized that a mechanism of shared risk is due to abnormal gene regulation in radial glia and interneurons during mid-gestation. Shared risk loci in FMRP targets, CHD5, CHD8, SCN2A, and neurexin 1 (NRXN1) are implicated in shared phenotypes of ASD, intellectual disability (ID), schizophrenia [Issifov., et al. 2014; Wang., et al. 2019]. Increased incidence of de novo pathogenic variants in periodic circadian regulator 1 (PER1) and lysine methyltransferase 2C (KMT2C) was found to be related to commonalities across ASD, ID, and bipolar disorder [Wang., et al. 2019]. Due to sharing of risk loci [Pinto., et al. 2010; McCarthy., et al. 2014], ASD and ID is found to coexist in $45 \%$ cases [3]. 
'Second-hit' to 23 susceptible gene loci including dopamine receptor D2 (DRD2), cholinergic receptor nicotinic alpha 7 subunit (CHRNA7), 5-hydroxytryptamine receptor 2A (HTR2A), solute carrier family 6 member 3 (SLC6A3), and tryptophan hydroxylase 2 (TPH2) in relation to dopamine and serotonin homeostasis was considered as a possible mechanism of shared features in ASD, bipolar disorder, and schizophrenia [27].

Increased prevalence of childhood autism in offspring of schizophrenic parents, and also early onset schizophrenia in autistic children as reposted by Rapoport., et al. (2009) and Sullivan., et al. (2012) [28,29], were supported by The Autism Spectrum Disorders Working Group of The Psychiatric Genomics Consortium (2017) [30] with the findings of shared risk loci including several genes involved in neurodevelopment, e.g. forkhead box P1 (FOXP1), exostosin glycosyltransferase 1 (EXT1), astrotactin 2 (ASTN2), monoADP ribosylase 2 (MACROD2), and histone deacetylase 4 (HDAC4).

There are high degree overlaps between autistic traits and ADHD (OR AD/HD) probrands [31], also sharing of susceptible gene loci like 7q36, 16p13,18p11, 15q24, and 12q24 [Nijmeijer., et al. 2010], and sharing of gene loci for nicotinic receptor signalling pathway and cell division [Martin., et al. 2014].

Although Angelman disease occurs due to deletion of and autism due to duplication of ubiquitin ligase gene UBE3A, there may be coexistence of both the disorder in the same person [Peters., et al. 2004; Williams., et al. 2010; Smith., et al. 2011; Kalsner., et al. 2015; Yi., et al. 2015].

The shared disease mechanism is yet to be perceived due to the complexities in interaction and overlap between neuro-psychiatric disorders.

\section{Modifying factors in ASD \\ Genetic factors}

Despite extensive studies, including CNVs and SNPs, to find specific genetic loci behind autism nothing conclusive has yet been achieved, probably due to effect of some second modifiers either at the somatic cell levels through 'Two-hit mechanism' like retinoblastomas proposed by Knudson, or by 'two-locus model' at germline level, as in Hirschprung disease [Knudson, 1971; Fisher., et al. 1994; McCallion., et al. 2003].
Artuso., et al. (2011) used array-hybridization technique to study genome of 8 cases of monogenic Rett syndrome of ASD, and identified 15 "likely" and 14 "unlikely" modulators of RTT phenotypes. Although, in CNVs or epigenetic regulation in discordant monozygotic twins revealed potential alterations in methylation patterns in a case and anomalies in another at 2 p25.3 region [Bruder., et al. 2008; Kunio., et al. 2013; Rio., et al. 2013], study with 100 twins failed to reveal discordant phenotype through CNV [Stamouli., et al. 2018]. Finding SHANK2 pathogenic variants in individuals with/without neuropsychiatric disease suggested the presence of additional variants in causing disease. Three cases with de novo SHANK2 mutations having deletions of CHRNA7 and cytoplasmic FMR1 interacting protein 1 (CYFIP1) - implicated in ASD - supported a "multiple-hit" model of autism [Leblond., et al. 2012].

An analysis revealed that 19 out of 20226 patients with CNVs in contact in 6 (CNTN6), a probable gene involved in ASD and other neurodevelopmental disorders [Repnikova., et al. 2019]. But, CNTN6 was found to have inconclusive role in causation of ASD. Specific 16p12.1 micro-deletion had a less severe phenotype in comparison to random second variants [Girirajan., et al. 2010]; and 22q11.2 deletion syndrome - all haploinsufficient for an mGluR network gene - found that $20 \%$, co-diagnosed with autism, had second-hit pathogenic variants [Wenger., et al. 2016]. Bonnet-Brilhault., et al. (2016) assessed a family with ID and ASD due to NLGN4X pathogenic variants, and found individuals with naive ASD having second-hit variants in glycine receptor beta (GRLB) and ankyrin 3 (ANK3).

Continuing advancements in sequencing technology, analyzing software and expansion of databases is laying the foundation to noteworthy developments in the study of genetic modifiers.

\section{Epigenetic and environmental factors}

Currently $40 \%-80 \%$ of autism cases are related to some risk genes, but epigenetic, particularly environmental modifiers, like advanced age of father, gestational complications and infections, or prenatal exposure to anti-convulsants, are playing prime roles in heterogeneity of manifestations [32]. Among the anti-convulsants, Valproate has been studied most, and thought to be a gene modifier by inhibiting histone deacetylase to cause autism [Kataoka., et 
al. 2013], through apoptotic cell death in the neocortex, decreased proliferation in the ganglionic eminence, increased homeobox A1 (HOXA1) expression, abnormal serotonergic differentiation via Achaete-Scute family BHLH transcription factor 1 (ASCL1) silencing, disrupted serotonin homeostasis in the amygdala, dendritic spine loss, reduced prefrontal dopaminergic activity, and disruption of the glutamatergic/GABAergic balance [33].

Impact on the transcriptome of an organism is based on epigenetic link between environmental risk factors and genetic susceptibility might be explored through more GWAS to understand the common ASD epigenomes.

Pro-inflammatory states involving cytokines and interleukins and also microglia could have some physiological and metabolic aetiogenesis of ASD $[34,35]$. Even dysbiosis of intestinal microbiome is also considered to alter immune function, leading to physiologic impact on learning, memory and behaviour; ultimately to ASD risk [Cao., et al. 2013; Bilbo., et al. 2015; Zhang., et al. 2015]. Beside cytokine related immune disruption, phosphocreatine-related mitochondrial dysfunction are having positive correlation with the severity of ASD manifestations [35]. Although causal role of such association are yet to be accertained [36].

Many of the recent studies suggest equal contribution of both genetic and non-shared environmental factors in aetiology of ASD [Roland., et al. 2011; Sandin., et al. 2014; Colvert., et al. 2015]. Smoking, alcohol intake, intake of valproate and SSRIs, exposure to environmental chemicals, like pesticides, metals, bisphenol A, during gestational period are considered to be major exogenous environmental factors leading to epigenetic changes through altered DNAm or DNMT expression/activity in the aetiogenesis of ASD [3741]. But researchers found it extremely difficult in accurately estimating the levels and timings of exposure in the mother and more critically and the foetus [36]. Among the endogenous environmental factors, major contributors are considered to be paternal age, might be maternal age also, preterm birth, maternal infections and autoimmune disorders, gestational diabetes mellitus (GDM), assisted reproductive technologies (ARTs), hypoxia-related obstetrics complications. Besides this, maternal stress and maternal nutrition are also considered to be important in the aetiology of ASD. Although there are some biologically plausible explanations behind these propositions, but the role of these factors are yet to be ascer- tained precisely [36]. Preterm labour, multiple births are found to be associated with significant gain of methylation (GOM) in OXTR. Also some epigenetic changes are noted in OXTR, SHANK3, BCL2, apoptosis regulator (BCL2) and RORA through altered DNAm; and hence are related to ASD cases [Leavey., et al. 2013; Atladottir., et al. 2016]. ART (IVF, ICSI) are related to preterm labour, multiple births, low birth weight, and all of these factors are independently found to be related to ASD $[42,43]$. Loss of methylation (LOM) is found to be in increased frequency subsequent to application of ART, which goes against the proposition of increased ASD cases following ART $[44,45]$. Hence, contribution of ART in causation of ASD is controversial [Conti., et al. 2013; Schieve., et al. 2015]. Paternal age is related to de-novo mutations in ASD probands at an escalated number; [Sanders., et al. 2012] and DNAm alterations are observed both in sperms and oocytes [Ge., et al. 2015; Milekic., et al. 2015]. Hypoxia-related obstetrics complications have significant toll on ASD risks with effect estimate more than 1.4 [46]. Infections during pregnancy [Atladottir., et al. 2016; Brown., et al. 2012; Lee., et al. 2015] and autoimmune disorders [Chen., et al. 2016] are having Odds Ratios of $1.24-1.37$ and 1.34 respectively related to increased prevalence of ASD. Maternal obesity and GDM have increased epigenetic alteration of DNAm and hence are considered to be related to causation of ASD, but no convincing data are there to validate such claims $[36,47,48]$. Maternal stress, assessed through general and social communication scores, were found to be associated with altered DNAm of OXTR, a recurring ASD-risk gene of interest [Rijlaarsdam., et al. 2016]. But, specific GxE interaction failed to confirm such association [36]. Poor maternal nutrition is also considered to be of paramount importance, and it is supported by the fact that folate supplementation helps in reducing the methylation of particular DNA through OCM-SAM pathway; and thus attenuating the ASD risk by OR: 0.61 [Schmidt., et al. 2012; Schmidt., et al. 2013; Suren., et al. 2013].

Studies with large sample sizes are required to elicit reflection of ASD-environment association of stable epigenomes detectable in foetus and neonates, and specific GxE correlation.

\section{Sex-linked factors}

ASD is said to affect males more than females, probably due to more external manifestations in male (aggression or increased repetitive behaviour vs. depression and avoiding demands), likely 
being influenced by hormones or genetics [49]. Autism in male manifests at a lower mutational burden [50]; even mothers of autistic boys have more mutation burden, particularly in 15q1113 duplication [Cook., et al. 1997; Schroer., et al. 1998; Gurrieri., et al. 1999; Boyar., et al. 2001] related to $\mathrm{GABA}_{\mathrm{A}}$ receptor causing perturbation of GABA signalling in autism [Al-Otaish., et al. 2018]. Protective action of oestrogen [Macrì., et al. 2010; Hoffman., et al. 2016], male predominant expression of chromatin regulation and immune response [51], male-specific down-regulation of $M e C P 2$ leading to abnormal glutamate activity [Kim., et al. 2016] might explain male vulnerability to, if not protection in female from, autism.

Reduced aromatase CYP19A1 activity leading to less conversion of testosterone to oestrogen in adolescent brain of ASD patients [Sarachana., et al. 2011; Crider., et al. 2014] might be related to diminished RAR activity involving RAR-related orphan receptor A (RORA) gene in susceptible male with autism [Nguyen., et al. 2010; Sarachana., et al. 2011] by more sex-dependent dysregulation of RORA gene [Hu., et al. 2015].

Transcriptomes, with enriched ECM molecules, in female mice might have a protective effect. Sex hormone probably impart divergent modulation [52] in sex-related ASD pathology [Estes., et al. 2015; Koyama., et al. 2015; Kim., et al. 2017; McCarthy., et al. 2017; Nadeem., et al. 2019] through their contrasting effect on immune system [53]. Analyzing biomarkers from individuals with Asperger's syndrome, Schwarz., et al. (2011) found 24 male-specific and 17 female-specific hits, including many immune-related molecules. Testosterone [Hatanaka., et al. 2015] also affects spine density, another phenotype strongly implicated in autism [54,55].

Key neurotransmitters, implicated in ASD, like GABA, glutamate, serotonin, and BDNF are modulated by sex hormones [Kim., et al. 2016; Saghazade., et al. 2017; Al-Otaish., et al. 2018; Edwards., et al. 2018; Ferri., et al. 2018; Garbarino., et al. 2018; Zieminska., et al. 2018].

Whether sexually dimorphic phenotypes in ASD are modulated by sex hormones or by some other modifiers, are yet to be established through intensive studies in the field.

\section{Conclusion}

After having an intensive sojourn through all the works by various scientists across the globe over last 75 years, we have identified a number of chromosomes, multitudes of gene loci, many genetic overlaps, and myriads of modifiers - endocrinal, environmental, and epigenetic. But, all these cannot be funnelled through a single common pathway, or even a specified channel, related to the causation of autism or even ASDs. More intensive studies using more intricate tools, even yet to come out, will have to be undertaken to cognize the arena of genetic background behind ASD.

But, the studies up till now are worthy enough. Through these studies we could identify susceptible and risk genes, their metabolic implications and possible therapeutic approaches to address those, either in ameliorating the manifestations or in preventing the occurrence of autism or ASDs. A lot more studies are sought for to enrich our knowledge in pharmacogenetics related to ASD.

Last, but not the least, to visit www.thetransporters.com which helps children to recognize emotions. Baron-Cohen and his Cambridge University colleagues collaborated with Britain>s National Autistic Society and a film production company created an animated series of vehicles for children, usually fond of toy carriages. In such a manner, toy trains, trams, trucks with human faces could display various facial expressions appropriately to the situation. Watching these programs for a long time at their bedrooms, autistic children gradually got tuned up with these expressions. With such intervention over a tangible period of time, autistic children could even react to different expressions like their non-autistic peers in the schools. This implies that specially designed training programs can induce social connectivity among the autistic children. Simultaneously, it raises a pertinent question; is autism or ASD is a special type of psycho-social developmental delay? And this question evokes another possibility of explaining autism as a phenomenon of delay in mirror neuron development.

To conclude, it can be said that genetics is highly implicated in, but not solely related to the causation of autism.

\section{Glossary}

(Table 1) 


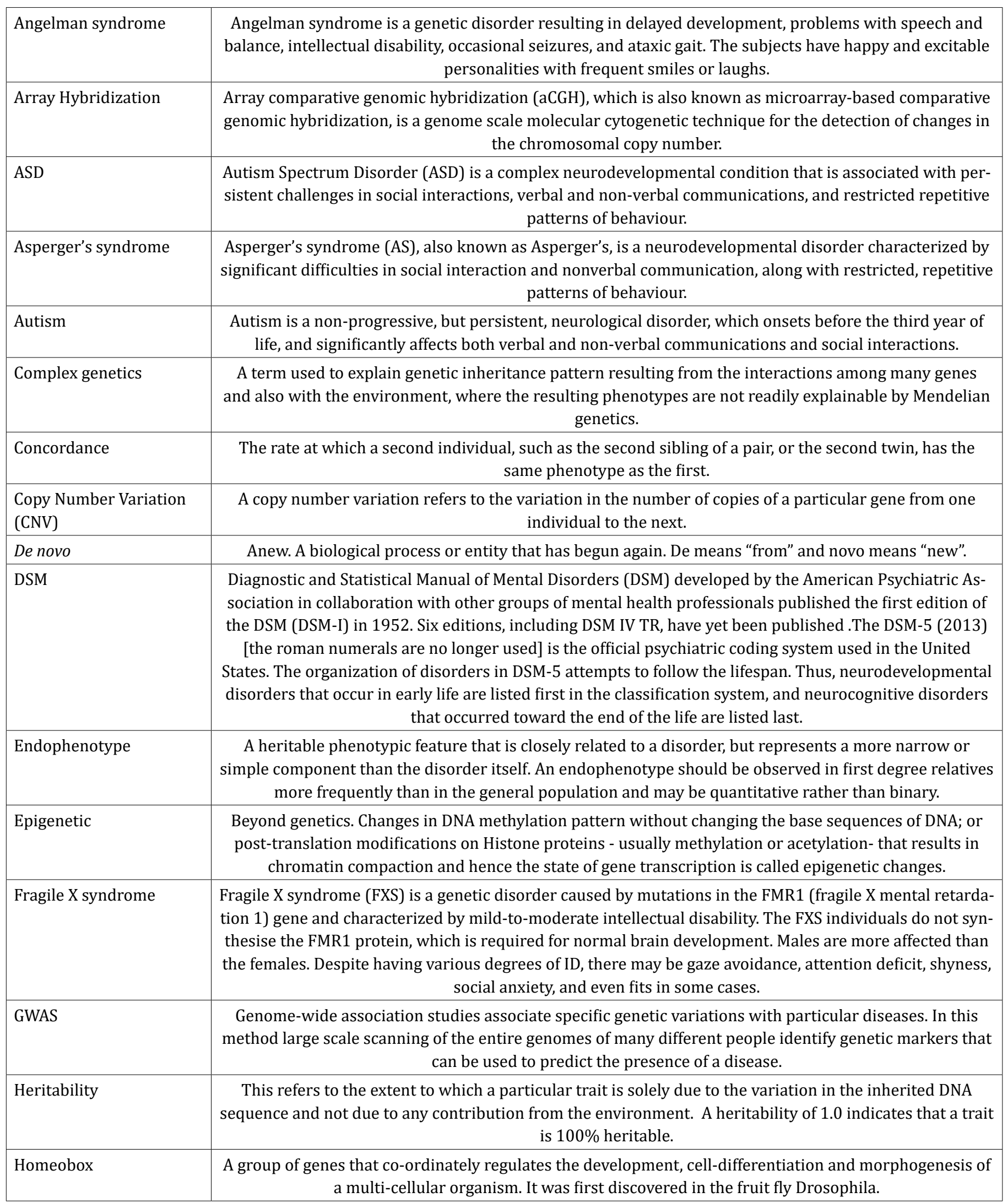




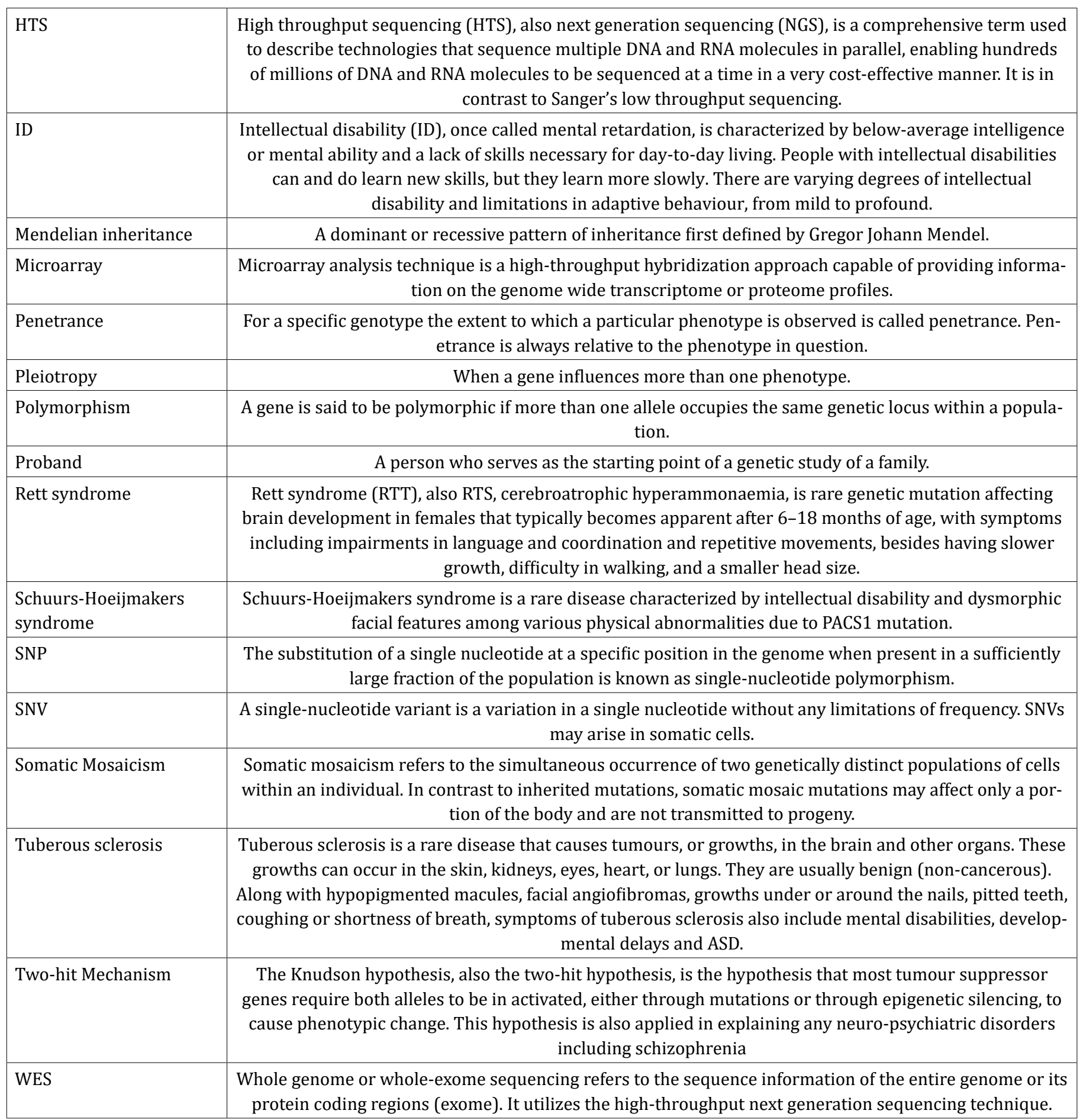

Table 1

Citation: Sukanti Bhattacharyya., et al. "Is Genetics Implicated in the Causation of Autism?". Acta Scientific Medical Sciences 6.3 (2022): 60-71. 


\section{Acknowledgement}

The authors, hereby, acknowledge the contributions made by Dr. Mrinal Kanti Bhattacharyya (Professor, Department of Biochemistry, School of Life Sciences, University of Hyderabad, Hyderabad Central University Rd, CUC, Gachibowli, Telangana 500046, India) and Dr. Damodar Prasad Goswami (Professor, Department of Mathematics, Netaji Subhash Engineering College, Panchpota, Garia, Kolkata, West Bengal 700152, India) in gathering knowledge on the matter, accumulating research articles, formulating the base work and in holistic development of the review article.

\section{Conflict of Interest}

The authors had no monetary interest or conflict of interest while developing this review article.

\section{Bibliography}

1. Maenner MJ., et al. "Prevalence of Autism Spectrum Disorder Among Children Aged 8 Years - Autism and Developmental Disabilities Monitoring Network, 11 Sites, United States, 2016". (MMWR) Surveillance Summaries 69 (2020): 1-12.

2. Loomes R., et al. "What is the male-to-female ratio in autism spectrum disorder? A systematic review and meta-analysis". Journal of the American Academy of Child and Adolescent Psychiatry 56 (2017): 466-474.

3. Lai MC., et al. "Autism". Lancet 383 (2014): 896-910.

4. Kanner L. "Autistic disturbances of affective contact". Nervous Child 2 (1943): 217-250.

5. Chaste P and Leboyer M. "Autism risk factors: genes, environment, and gene-environment interactions". Dialogues in Clinical Neuroscience 14 (2012): 281-292.

6. Constantino J N., et al. "Sibling recurrence and the genetic epidemiology of autism". The American Journal of Psychiatry 167 (2010): 1349-1356.

7. Risch N., et al. "Familial recurrence of autism spectrum disorder: evaluating genetic and environmental contributions". The American Journal of Psychiatry 171 (2014): 1206-1213.

8. Sandin S., et al. "The familial risk of autism". JAMA 311 (2014): 1770-1777.

9. IMGSAC. "Full genome screen for autism with evidence for linkage to a region on chromosome $7 \mathrm{q} \mid$ human molecular genetics | oxford academic". Human Molecular Genetics 7 (1998): 571-578.
10. International Molecular Genetic Study of Autism Consortium [IMGSAC]. "A genomewide screen for autism: strong evidence for linkage to chromosomes 2q, 7q, and 16p". American Journal of Human Genetics 69 (2001): 570-581.

11. Stern D., et al. "Association of the missense variant p.Arg203Trp in PACS1 as a cause of intellectual disability and seizures". Clinical Genetics 92 (2017): 221-223.

12. Woodbury-Smith M., et al. "Progress in the genetics of autism spectrum disorder". Developmental Medicine and Child Neurology 60 (2018): 445-451.

13. Schmunk G and Gargus J J. "Channelopathy pathogenesis in autism spectrum disorders”. Frontiers in Genetics 4 (2013): 222.

14. Giovedí S., et al. "Involvement of synaptic genes in the pathogenesis of autism spectrum disorders: the case of synapsins". Frontiers in Pediatrics 2 (2014): 94.

15. Stessman H A F., et al. "Targeted sequencing identifies 91 neurodevelopmental disorder risk genes with autism and developmental disability biases". Nature Genetics 49 (2017): 515526.

16. De Rubeis S., et al. "Synaptic, transcriptional and chromatin genes disrupted in autism". Nature 515 (2014): 209-215.

17. Lim ET., et al. "Rates, distribution and implications of postzygotic mosaic mutations in autism spectrum disorder". Nature Neuroscience 20 (2017): 1217-1224.

18. Ronemus M., et al. "The role of de novo mutations in the genetics of autism spectrum disorders". Nature Reviews Genetics 15 (2014): 133-141.

19. Gilissen C., et al. "Genome sequencing identifies major causes of severe intellectual disability". Nature 511 (2014): 344-347.

20. Pizzo L., et al. "Rare variants in the genetic background modulate cognitive and developmental phenotypes in individuals carrying disease-associated variants". Genetic Medicine 21 (2016) 91:6-825.

21. The Autism Genome Project Consortium P., et al. "Mapping autism risk loci using genetic linkage and chromosomal rearrangements". Nature Genetics 39 (2007): 319-328.

22. Duffney L J., et al. "Epigenetics and autism spectrum disorder: a report of an autism case with mutation in $\mathrm{H} 1$ linker histone HIST1H1E and literature review". American Journal of Medical Genetics 177 (2018): 426-433. 
23. Kubota T andMochizuki K. "Epigenetic effect of environmental factors on autism spectrum disorders". International Journal of Environmental Research and Public Health 13 (2016): E504.

24. Cross-Disorder Group of the Psychiatric Genomics Consortium. "Identification of risk loci with shared effects on five major psychiatric disorders: a genome-wide analysis". Lancet 381 (2013): 1371-1379.

25. Cross-Disorder Group of the Psychiatric Genomics Consortium Lee SH., et al. "Genetic relationship between five psychiatric disorders estimated from genome-wide SNPs". Nature Genetics 45 (2013): 984-994.

26. Schork A J., et al. "A genome-wide association study of shared risk across psychiatric disorders implicates gene regulation during fetal neurodevelopment". Nature Neuroscience 22 (2019): 353-361.

27. Khanzada N S., et al. "Gene Analytics pathway analysis and genetic overlap among autism spectrum disorder, bipolar disorder and schizophrenia". International Journal of Molecular Sciences 18 (2017): E527.

28. Rapoport J., et al. "Autism spectrum disorders and childhoodonset schizophrenia: clinical and biological contributions to a relation revisited". Journal of the American Academy of Child and Adolescent Psychiatry 48 (2009): 10-18.

29. Sullivan P F., et al. "Family history of schizophrenia and bipolar disorder as risk factors for autism". Archives of General Psychiatry 69 (2012): 1099-1103.

30. The Autism Spectrum Disorders Working Group of The Psychiatric Genomics Consortium. "Meta-analysis of GWAS of over 16,000 individuals with autism spectrum disorder highlights a novel locus at 10q24.32 and a significant overlap with schizophrenia". Molecular Autism 8 (2017): 21.

31. Stergiakouli E., et al. "Shared genetic influences between dimensional ASD and ADHD symptoms during child and adolescent development". Molecular Autism 8 (2017): 18.

32. Ohkawara T., et al. "Maternal viral infection during pregnancy impairs development of fetal serotonergic neurons". Brain Development 37 (2015): 88-93.

33. Dufour-Rainfray D., et al. "Behavior and serotonergic disorders in rats exposed prenatally to valproate: a model for autism". Neuroscience Letter 470 (2010): 55-59.

34. Nardone $\mathrm{S}$ and Elliott $\mathrm{E}$. "The interaction between the immune system and epigenetics in the etiology of Autism Spectrum disorders". Frontiers in Neuroscience 10 (2016): 329.
35. Rossignol DA and Frye RE. "A review of research trends in physiological abnormalities in autism spectrum disorders: immune dysregulation, inflammation, oxidative stress, mitochondrial dysfunction and environmental toxicant exposures". Molecular Psychiatry 17.4 (2012): 389-401.

36. Siu MT and Weksberg R. "Epigenetics of Autism Spectrum Disorder". Advances in Experimental Medicine and Biology 978 (2017): 63-90.

37. Dufour-Rainfray D., et al. "Fetal exposure to teratogens: evidence of genes involved in autism". Neuroscience and Biobehavioral Reviews 35.5 (2011): 1254-1265.

38. Kim YS and Leventhal BL. "Genetic epidemiology and insights into interactive genetic and environmental effects in autism spectrum disorders". Biological Psychiatry 77.1 (2015): 66-74.

39. Lee KW., et al. "Prenatal exposure to maternal cigarette smoking and DNA methylation: epigenome-wide association in a discovery sample of adolescents and replication in an independent cohort at birth through 17 years of age". Environmental Health Perspectives 123.2 (2015): 193-199.

40. Roullet FI., et al. "In utero exposure to valproic acid and autism-a current review of clinical and animal studies". Neurotoxicology and Teratology 36 (2013): 47-56.

41. Yaoi T., et al. "Genome-wide analysis of epigenomic alterations in fetal mouse forebrain after exposure to low doses of bisphenol A". Biochemical and Biophysical Research Communications 376.3 (2008): 563-567.

42. Gardener H., et al. "Perinatal and neonatal risk factors for autism: a comprehensive meta-analysis". Pediatrics 128.2 (2011): 344-355.

43. Grafodatskaya D., et al. "The health risks of ART". EMBO Report 14.2 (2013): 129-135.

44. DeBaun MR., et al. "Association of in vitro fertilization with Beckwith-Wiedemann syndrome and epigenetic alterations of LIT1 and H19". American Journal of Human Genetics 72.1 (2003): 156-160.

45. Sutcliffe AG., et al. "Assisted reproductive therapies and imprinting disorders-a preliminary British survey". Human Reproduction 21.4 (2006): 1009-1011.

46. Froehlich-Santino W., et al. "Prenatal and perinatal risk factors in a twin study of autism spectrum disorders". Journal of Psychiatric Research 54 (2014): 100-108. 
47. Krakowiak P., et al. "Autism-specific maternal anti-fetal brain autoantibodies are associated with metabolic conditions". Autism Research 10.1 (2016): 89-98.

48. Li YM., et al. "Association between maternal obesity and autism spectrum disorder in offspring: a meta-analysis". Journal of Autism and Developmental Disorders 46.1 (2016): 95-102.

49. Werling D M., et al. "Sex differences in autism spectrum disorders". Current Opinion in Neurology 26 (2013): 146-153.

50. Desachy G., et al. "Increased female autosomal burden of rare copy number variants in human populations and in autism families". Molecular Psychiatry 20 (2015): 170-175.

51. Ziats M N and Rennert O M. "Sex-biased gene expression in the developing brain: implications for autism spectrum disorders". Molecular Autism 4 (2013): 10.

52. Baron-Cohen S., et al. "Sex differences in the brain: implications for explaining autism". Science 310 (2005): 819-823.

53. Roved J., et al. "Sex differences in immune responses: hormonal effects, antagonistic selection, and evolutionary consequences". Hormones and Behavior 88 (2017): 95-105.

54. Liu S., et al. "A rare variant identified within the GluN2B CTerminus in a patient with autism affects NMDA receptor surface expression and spine density". Journal of Neuroscience 37 (2017b): 4093-4102.

55. Liu X., et al. "Idiopathic autism: cellular and molecular phenotypes in pluripotent stem cell-derived neurons". Molecular Neurobiology 54 (2017b): 4507-4523.

56. Rylaarsdam Lauren and Alicia Guemez-Gamboa. "Genetic Causes and Modifiers of Autism Spectrum Disorder". Frontiers in Cellular Neuroscience 13 (2019): 385.

\section{Assets from publication with us}

- Prompt Acknowledgement after receiving the article

- Thorough Double blinded peer review

- Rapid Publication

- Issue of Publication Certificate

- High visibility of your Published work

Website: www.actascientific.com/

Submit Article: www.actascientific.com/submission.php

Email us: editor@actascientific.com

Contact us: +919182824667 